\title{
Correlation of a strong lunar magnetic anomaly with a high-albedo region of the Descartes mountains
}

\author{
N. C. Richmond, ${ }^{1}$ L. L. Hood, ${ }^{1}$ J. S. Halekas, ${ }^{2}$ D. L. Mitchell, ${ }^{2}$ R. P. Lin, ${ }^{3}$ M. Acuña, ${ }^{3}$ \\ and A. B. Binder ${ }^{4}$ \\ Received 16 January 2003; revised 3 March 2003; accepted 7 March 2003; published 9 April 2003
}

[1] Mapping and model simulations of Lunar Prospector magnetometer measurements show that the source of the strongest known magnetic anomaly on the lunar near side (42 nanoTeslas at $18.6 \mathrm{~km}$ altitude) coincides approximately with a high-albedo region of the Descartes mountains centered $60 \mathrm{~km}$ south-southeast of the Apollo 16 landing site. The Descartes mountains represent primary ejecta from one or more basin-forming events (Imbrium and/or Nectaris), supporting the hypothesis that basin ejecta materials emplaced $>3.8$ Gyr ago are the main sources of lunar magnetic anomalies. The higher albedo of the surface at this location is consistent with a significant role for solar wind ions in the optical maturation (or "space weathering") of the lunar surface. INDEX TERMS: 5440 Planetology: Solid Surface Planets: Magnetic fields and magnetism; 5470 Planetology: Solid Surface Planets: Surface materials and properties; 6250 Planetology: Solar System Objects: Moon (1221). Citation: Richmond, N. C., L. L. Hood, J. S. Halekas, D. L. Mitchell, R. P. Lin, M. Acuña, and A. B. Binder, Correlation of a strong lunar magnetic anomaly with a high-albedo region of the Descartes mountains, Geophys. Res. Lett., 30(7), 1395, doi:10.1029/2003GL016938, 2003.

\section{Introduction}

[2] Since the years following the Apollo manned lunar landing program, it has been known that several relatively strong and isolated lunar magnetic anomalies correlate with unusual, curvilinear albedo markings; the most well-known example is the Reiner Gamma magnetic anomaly and albedo marking on western Oceanus Procellarum (Hood et al. [2001] and references therein). Theoretical models for the interaction of the solar wind with lunar magnetic anomalies have indicated that these strongest anomalies should be capable of significantly deflecting the ion bombardment, producing local shielded areas at the lunar surface (e.g., Harnett and Winglee [2000]). It has therefore been proposed that the observed correlation of strong magnetic anomalies with high-albedo surface markings may represent macroscopic evidence for a significant role of the solar wind ion bombardment in the space weathering of the lunar surface and, by extension, that of other airless USA.

${ }^{1}$ Lunar and Planetary Laboratory, University of Arizona, Tucson, AZ, USA.

${ }^{2}$ Space Sciences Laboratory, University of California, Berkeley, CA,

${ }^{3}$ Goddard Space Flight Center, Greenbelt, MD, USA.

${ }^{4}$ Lunar Research Institute, Tucson, AZ, USA.

Copyright 2003 by the American Geophysical Union. 0094-8276/03/2003GL016938 silicate surfaces in the inner solar system [Hood and Schubert, 1980; Hood and Williams, 1989].

[3] However, the geologic origin of these strong magnetic anomalies and their associated albedo markings has been controversial. According to one hypothesis, the albedo markings are a result of surface scouring (surficial mass removal) by relatively recent $(<1 \mathrm{Myr}$ old $)$ cometary coma impacts; the associated magnetic anomaly sources are then suggested to be surficial materials heated and exposed to transient magnetic fields during the coma impacts [Schultz and Srnka, 1980]. Detailed spectral reflectance studies of the Reiner Gamma albedo feature have been unable to eliminate the cometary impact model ([Bell and Hawke, 1987] and references therein).

[4] Alternatively, it has been proposed that the sources of most strong lunar magnetic anomalies consist of basin impact ejecta containing unusual abundances of metallic iron remanence carriers [Strangway et al., 1973; Hood et al., 2001]. These ejecta materials may have been magnetized by a temporary lunar core dynamo field (for a review, see Fuller and Cisowski [1987]) and/or by transient fields generated by impact-produced plasmas [Hood and Vickery, 1984; Crawford and Schultz, 1991]. The associated albedo markings are then attributed to the solar wind deflection mechanism (e.g., Hood and Williams [1989]). Distinguishing between these models has been difficult. For example, the source of the strongest anomaly known previously on the near side, the Reiner Gamma anomaly, remains unclear. The visible surface consists of a thin veneer of mare basalt flows that obscures the underlying terrain.

[5] Here we report mapping and interpretation of a strong, isolated magnetic field anomaly on the central lunar near side using Lunar Prospector magnetometer (MAG) data obtained at low altitudes during 1999. This anomaly was first identified using Lunar Prospector electron reflectometer (ER) data [Halekas et al., 2001] and was shown to correlate approximately with the Descartes mountains near the Apollo 16 landing site. Independent analysis of the magnetometer data allowed identification of a small number of relatively undisturbed MAG orbits with coverage over most of this anomaly.

\section{Data Analysis and Modeling}

[6] Figure 1 shows the field magnitude in nT along a series of four orbits, which occurred on April 13, 1999, at an altitude of approximately $18.6 \mathrm{~km}$. The field magnitude is plotted as a function of latitude; the longitude of the orbit at $10^{\circ} \mathrm{S}$ is also indicated. The lowermost orbit on the plot (near $15.7^{\circ} \mathrm{E}$ longitude) passes within $6 \mathrm{~km}$ of the Apollo 16 landing site (indicated by the arrow). A maximum field 
Day 103, 1999, orbits: $345-348$

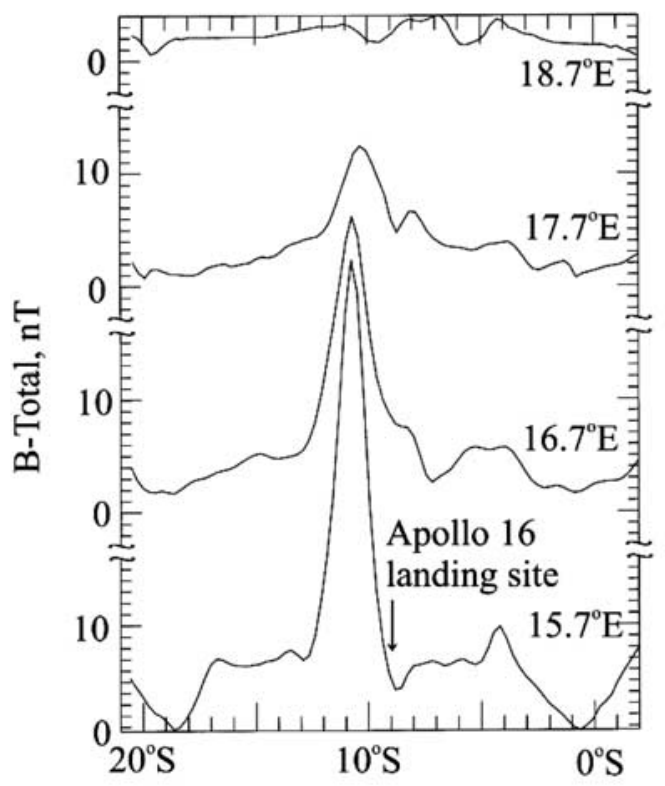

Figure 1. Field magnitude measured at the spacecraft altitude $(\sim 18.6 \mathrm{~km})$ plotted as a function of latitude along a series of orbits over the Descartes anomaly. The longitude of each orbit at $10^{\circ} \mathrm{S}$ is given on the right side of the figure. The latitude of the Apollo 16 landing site along the lowermost orbit is also indicated.

magnitude of $\sim 42 \mathrm{nT}$ is reached near $10.7^{\circ} \mathrm{S}$ latitude (about $60 \mathrm{~km}$ south of the landing site).

[7] Continuation of the field magnitude to a common altitude $(18 \mathrm{nT}$ at $28 \mathrm{~km})$ and direct comparisons with other anomalies elsewhere on the Moon indicates that this anomaly is the largest in absolute magnitude of any single anomaly yet mapped on the lunar near side. For comparison, the Reiner Gamma anomaly has a magnitude of about $15 \mathrm{nT}$ at $28 \mathrm{~km}$ while the Rima Sirsalis anomaly [Halekas et al., 2001; Hood et al., 2001] has an amplitude of about $7 \mathrm{nT}$ at this altitude. On the far side, only one anomaly in the Crisium antipode group [Hood et al., 2001] is larger in absolute magnitude than this anomaly. Surface fields in the vicinities of these anomalies are likely to exceed $\sim 10^{3} \mathrm{nT}$ (e.g., Hood and Williams [1989]).

[8] Figure 2 is a superposition of a contour map of the two-dimensionally filtered field magnitude produced from the data of Figure 1 at a constant altitude of $19.0 \mathrm{~km}$ onto a photograph of the region. To construct the map using the available orbits with a 1 degree longitude spacing, three additional artificial orbits of data were first generated by linear interpolation at mean longitudes of $16.2^{\circ} \mathrm{E}, 17.2^{\circ} \mathrm{E}$, and $18.2^{\circ} \mathrm{E}$. Two-dimensional smoothing was then applied twice using a 5 by 5 moving boxcar filter with a size of $0.625^{\circ}$ by $0.625^{\circ}$. The effective spatial resolution of the map is $\sim 1.2$ degrees. The anomaly maximum (smoothed amplitude $\sim 30 \mathrm{nT}$ ) correlates most closely with the western side of the high-albedo portion of the southern Descartes mountains (labeled "DM"). Direct coverage is not available over the Cayley formation (labeled "CF"). The field magnitude at $19 \mathrm{~km}$ altitude directly above the Apollo 16 landing site, where small-scale surface fields exceeding $300 \mathrm{nT}$ were measured, is approximately $6 \mathrm{nT}$.
[9] In order to locate more accurately the probable source of the Descartes anomaly, the three vector field components were modeled using an iterative procedure. Specifically, because this anomaly is relatively isolated and dominantly dipolar, it is assumed for simplicity that the source consists of a thin circular disk located at or near the surface. The modeling procedure varies the disk location, radius, dipole moment per unit area (which is assumed to be constant across the disk), and the orientation of the mean magnetization vector in the disk, until a minimum-variance fit to the data is obtained. Both the location and the radius of the disk (but not the thickness) are constrained by the data. The model that produced a minimum variance had the following parameters. Magnetic dipole moment: 12,500 nT-km radius: $18 \mathrm{~km}$; center-of-disk location: $10.8^{\circ} \mathrm{S}, 15.8^{\circ} \mathrm{E}$; bulk magnetization vector: inclination $20^{\circ}$; declination $10^{\circ}$. As shown in Figure 3, despite limited coverage, the model describes the observations reasonably well. Comparison of the final model parameters with Figure 2 shows that the location and size of the disk coincides with the higheralbedo area of the mountains within the errors of the analysis. An examination of Lunar Prospector Gamma Ray Spectrometer Th and $\mathrm{FeO}$ data (see http://wufs.wustl. edu/missions/lunarp/reduced.htm) for the source location confirms that the surface composition of the high-albedo portion of the Descartes does not differ appreciably from that of neighboring regions. The source is therefore most probably at depth within the Descartes mountains rather than at the surface.

\section{Interpretation and Conclusions}

[10] The Descartes mountains and the Cayley formation are representative of two geologic units that are commonly

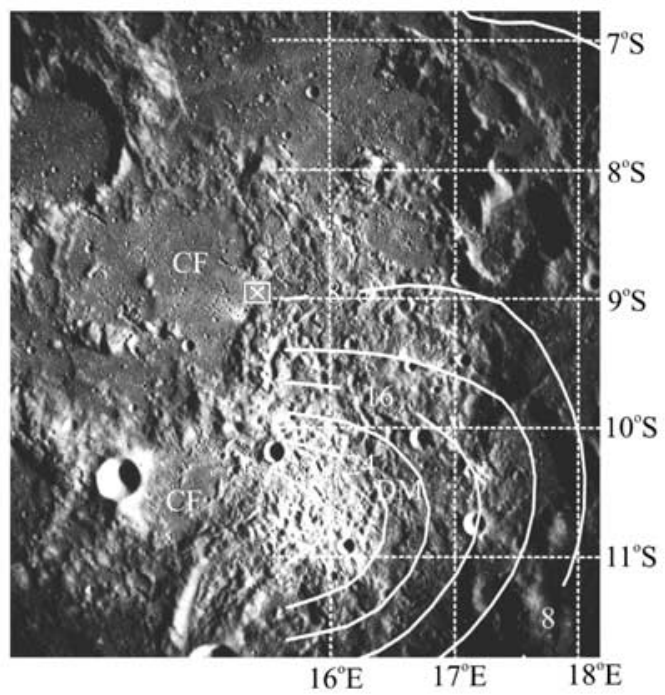

Figure 2. Contour map of the two-dimensionally filtered magnetic field magnitude (in nT) at an altitude of $19 \mathrm{~km}$ in the vicinity of the Apollo 16 landing site (boxed cross near center). The photograph is a portion of Apollo 16 mapping camera frame 0161. Several exposures of the Cayley formation $(\mathrm{CF})$ and the adjacent Descartes mountains (DM) are indicated. The $11-\mathrm{km}$ crater Dolland is at the lower left. 
A)

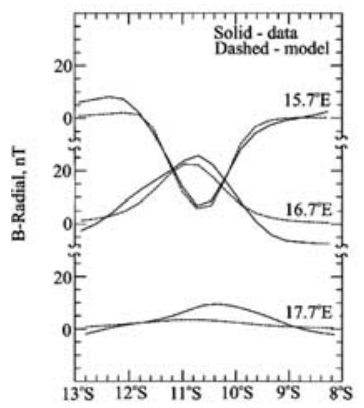

B)

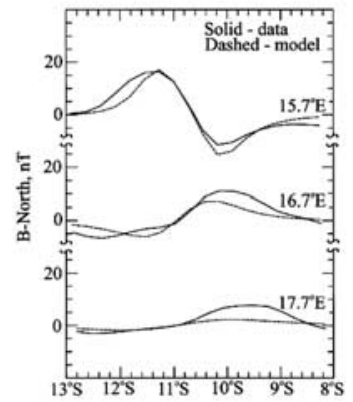

c)

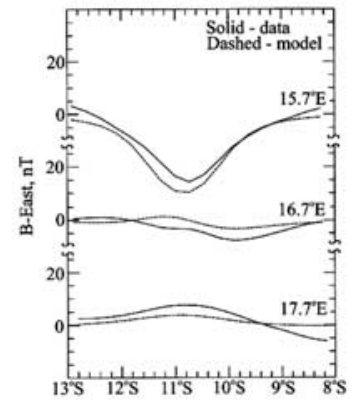

Figure 3. Comparison of observed and model-calculated field components along the lower three orbits of Figure 1. See the text.

found in the lunar highlands: hilly and furrowed terra and flat light plains, respectively. Prior to the Apollo 16 mission, a volcanic origin for these units was generally accepted. However, the return of heavily brecciated samples of impact origin from the vicinity of the landing site reversed this view and led to the currently accepted interpretation that these units represent primary and secondary basin ejecta materials [Wilhelms, 1984]. More specifically, it is generally agreed that the Descartes mountains represent primary basin ejecta although it remains uncertain whether the Imbrium impact or the Nectaris impact, or both, were responsible for its deposition [Hodges and Muehlberger, 1981; Spudis, 1984; Hawke and Head, 1980]. Therefore, the location of the Descartes magnetic anomaly source (Figure 2) represents new and more compelling evidence that relatively ancient ( $>3.8$ Gyr old) impact basin ejecta are the sources of the strongest magnetic anomalies on the Moon. Variable metallic iron contents and iron particle size ranges likely explain why some ejecta units are more magnetic than others.

[11] The conclusion that impact basin ejecta units are primary lunar magnetic anomaly sources is consistent with returned sample data and with surface magnetic field measurements acquired at the Apollo landing sites. The sample data show that the main ferromagnetic carriers in lunar materials, metallic iron particles in the single-domain size range, are common in impact-produced breccias and fines [Fuller and Cisowski, 1987]. Measured surface magnetic fields were generally weak at those Apollo landing sites that were dominated by mare basalt flows but were stronger at highland sites dominated by impact-generated materials [Dyal et al., 1974]. The strongest surface fields (up to $327 \mathrm{nT}$ ) at any landing site were measured along a surface traverse close to the Apollo 16 site in an area dominated by the Cayley formation and the adjacent Descartes mountains. Those Cayley plains materials near the Apollo 16 landing site were later shown to have unusually high abundances of metallic iron [Korotev, 1994]. These results led D. W. Strangway and others to propose originally that basin ejecta materials, such as the Cayley formation, are among the most strongly magnetized materials on the Moon [Strangway et al., 1973].

[12] As shown in Figure 4, the high-albedo section of the Descartes mountains that correlates with the strongest nearside magnetic anomaly is one of a number of similar bright areas in the central highlands that are most prominent near the time of full Moon. However, as is documented on standard USGS maps (e.g., Milton [1968]), other nearby bright regions (those labeled $\mathrm{A}, \mathrm{B}, \mathrm{C}, \mathrm{E}, \mathrm{F}$, and $\mathrm{G}$ ) invariably contain a large young (Copernican-aged) crater near their centers. An examination of Lunar Prospector ER data (see Plate 1 of ref. 13) and MAG data indicates that no significant magnetic anomaly is associated with A, B, C, E, $\mathrm{F}$, or G. In contrast, the Descartes albedo unit (D), which correlates with a strong magnetic anomaly, contains only one or two small young craters near one edge. The high albedos of A, B, C, E, F, and G can therefore be attributed to the presence of freshly exposed materials in the form of impact ejecta surrounding the craters. But the high albedo of the Descartes region (D) can not be so explained. Early interpretations of this albedo feature [Head and Goetz, 1972] suggested that it may represent evidence for Copernican-age volcanism. However, the observed strong mag-

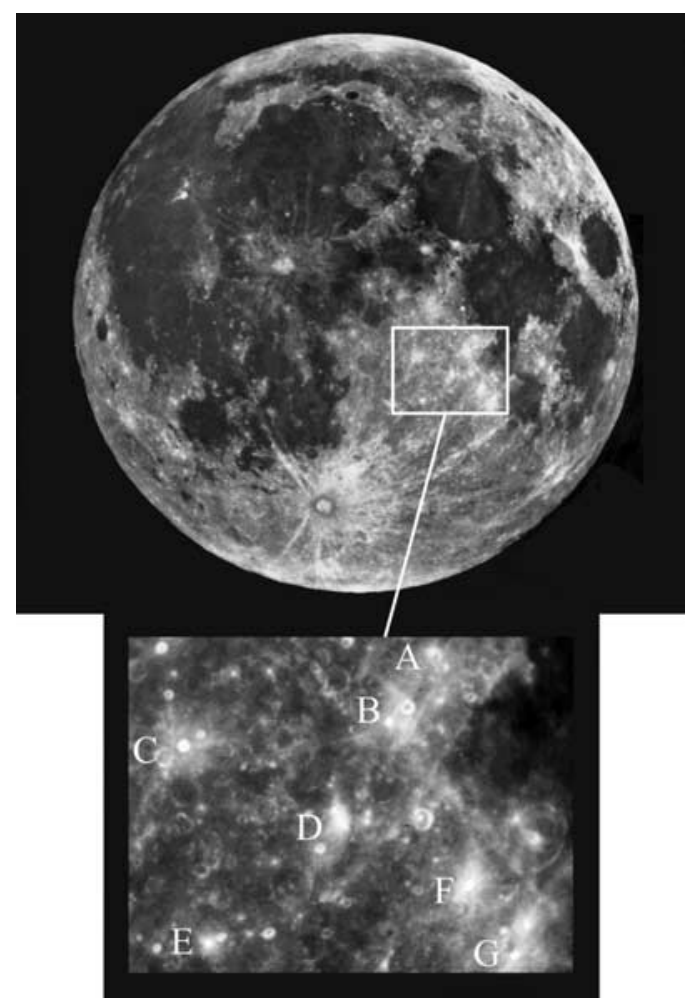

Figure 4. Enlarged section of an Earth-based telescopic photograph [Kuiper et al., 1967] of the full Moon showing a series of high-albedo regions on the east-central near side. 
netic anomaly is not consistent with this suggestion since volcanic materials are poorly magnetized elsewhere on the Moon. Since the Descartes mountains formed $>3.8$ Gyr ago, a possible interpretation is that some mechanism has preferentially maintained the original high albedo of the region against the space weathering process.

[13] Specifically, we consider that these data may represent new macroscopic evidence for a significant contribution of the solar wind ion bombardment to space weathering of airless silicate bodies in the solar system. Both micrometeoroid impacts and solar wind ion bombardment have previously been considered as likely external agents for producing the nanophase Fe that leads to "optical maturation" of lunar surface materials [Housley, 1977; Pieters et al., 1993; Keller et al., 1999]. However, soil studies alone have not yet established whether the solar wind ion bombardment is a necessary and significant component of the space weathering process [Taylor et al., 2001].

[14] As noted earlier, model calculations, including numerical magnetohydrodynamical simulations, strongly suggest that the highest-amplitude lunar anomalies are capable of deflecting the solar wind, producing local surface areas shielded from the ion bombardment. In contrast, although micrometeoroids exposed to solar radiation and space plasmas will acquire significant charge, their chargeto-mass ratios are too small to yield significant deflections in lunar fields. For example, for a break-up potential of 600 volts (e.g., Fechtig et al. [1979]), a micrometer sized particle would have a maximum possible charge-to-mass ratio of $\sim 3 \times 10^{-5}$ that of a proton. Assuming a surface field strength of $\sim 1000 \mathrm{nT}$, the gyroradius of a solar wind proton would be $\sim 4.2 \mathrm{~km}$ while that of a maximally charged micrometeoroid moving at $10 \mathrm{~km} / \mathrm{s}$ would be $\sim 3000 \mathrm{~km}$. Thus, while a solar wind proton would be easily deflected in a lunar field with scale size $\sim 10$ 's of km, a typical micrometeoroid would be nearly undeflected in such a field.

[15] Acknowledgments. We are grateful to G. T. DeLory of the University of California for discussions regarding the magnetometer data. Supported by NASA through a contract from the Lunar Research Institute.

\section{References}

Bell, J., and B. R. Hawke, Recent comet impacts on the Moon: The evidence from remote-sensing studies, Pub. Astron. Soc. Pacific, 99, $862-$ 867, 1987.

Crawford, D., and P. Schultz, Laboratory investigations of impact-generated plasma, J. Geophys. Res., 96, 18,807-18,819, 1991

Dyal, P., C. W. Parkin, and W. D. Daily, Magnetism and the Interior of the Moon, Rev. Geophys. Space Phys., 12, 568-591, 1974.

Fechtig, H., E. Grün, and G. Morfill, Micrometeoroids within ten Earth radii, Planet. Space Sci., 27, 511-531, 1979.

Fuller, M., and S. Cisowski, Lunar paleomagnetism, in Geomagnetism, edited by J. Jacobs, Academic Press, San Diego, CA, pp. 307-456, 1987.

Halekas, J. S., D. L. Mitchell, R. P. Lin, S. Frey, L. Hood, M. Acuña, and A. B. Binder, Mapping of lunar crustal magnetic fields using Lunar Prospector electron reflectometer data, J. Geophys. Res., 106, 27,841$27,852,2001$
Harnett, E., and R. Winglee, Two-dimensional MHD simulation of the solar wind interaction with magnetic field anomalies on the surface of the Moon, J. Geophys. Res., 105, 24,997-25,007, 2000.

Hawke, B. R., and J. W. Head, The distal deposits of lunar basins as exemplified by material collected at the Apollo 14 and 16 landing sites, in Papers Presented to the Conference on Multi-ring Basin Formation and Evolution, LPI Contribution 414, p. 36-38, Lunar and Planetary Institute, Houston, 1980.

Head, J. W., and A. F. H. Goetz, Descartes region: evidence for Copernicanage volcanism, J. Geophys. Res., 77, 1368-1374, 1972.

Hodges, C., and W. Muehlberger, K. A summary and critique of geologic hypotheses, in Geology of the Apollo 16 Area, Central Highlands (Geological Survey Prof. Paper 1048), edited by G. Ulrich, C. Hodges, and W. Muehlberger, U. S. Govt. Printing Office, Washington, D. C., pp. $215-230,1981$

Hood, L., and G. Schubert, Lunar magnetic anomalies and surface optical properties, Science, 208, 49-51, 1980

Hood, L., and A. Vickery, Magnetic field amplification and generation in hypervelocity meteoroid impacts with application to lunar paleomagnetism, Proc. Lunar Planet. Sci. Conf. 15th, Part 1, J. Geophys. Res., 89, C211-C223, 1984

Hood, L., and C. Williams, The lunar swirls: Distribution and possible origins, in Proc. Lunar Planet. Sci. Conf. 19th, edited by G. Ryder and V. Sharpton, Lunar Planet. Inst., Houston, TX., pp. 99-113, 1989.

Hood, L. L., A. Zakharian, J. Halekas, D. L. Mitchell, R. P. Lin, M. H. Acuña, and A. B. Binder, Initial mapping and interpretation of lunar crustal magnetic fields using Lunar Prospector magnetometer data, J. Geophys. Res., 106, 27,825-27,839, 2001.

Housley, R. M., Solar wind and micrometeorite effects in the lunar regolith, Philos. Trans. R. Soc. London, Ser. A, 285, 363-370, 1977.

Keller, L. P., S. J. Wentworth, D. S. McKay, L. A. Taylor, C. Pieters, and R. V. Morris, Space weathering in the fine size fractions of lunar soils: Soil maturity effects, in Workshop on New Views of the Moon II, Lunar and Planet. Inst., Houston, TX., 32-34, 1999.

Korotev, R., Compositional variation in Apollo 16 impact-melt breccias and inferences for the geology and bombardment history of the Central Highlands of the Moon, Geochim. Cosmochim. Acta, 58, 3931-3969, 1994.

Kuiper, G. P., E. A. Whitaker, R. G. Strom, J. W. Fountain, and S. M. Larson, Consolidated Lunar Atlas, Lunar and Planetary Laboratory, University of Arizona, Tucson, 1967.

Milton, D. J., Geologic Map of the Theophilus Quadrangle of the Moon, U. S. G. S. Map I-546; U. S. Geol. Surv., Washington D. C., 1968.

Pieters, C., E. Fischer, O. Rode, and A. Basu, Optical effects of space weathering: The role of the finest fraction, J. Geophys. Res., 98, 20,817-20,824, 1993

Schultz, P. H., and L. J. Srnka, Cometary collisions on the Moon and Mercury, Nature, 284, 22-26, 1980.

Spudis, P. D., Apollo 16 site geology and impact melts: Implications for the geologic history of the lunar highlands, Proc. 15th Lunar Planet. Sci. Conf. Part 1, J. Geophys. Res. Suppl., 89, C95-C107, 1984.

Strangway, D. W., H. Sharpe, W. Gose, and G. Pearce, Lunar magnetic anomalies and the Cayley Formation, Nature, 246, 112-114, 1973.

Taylor, L. A., C. M. Pieters, L. P. Keller, R. V. Morris, and D. S. McKay, Lunar mare soils: Space weathering and the major effects of surface-correlated nanophase Fe, J. Geophys. Res., 106, 27,98527,999, 2001

Wilhelms, D. E., Moon, in Geology of the Terrestrial Planets (NASA SP469), edited by M. Carr, R. S. Saunders, R. G. Strom, and D. Wilhelms, National Aeronautics and Space Administration, Washington, D. C., pp. $107-205,1984$

N. C. Richmond and L. L. Hood, Lunar and Planetary Laboratory, University of Arizona, 1629 E. University Blvd., Tucson, Arizona 85721, USA. (nic@lpl.arizona.edu; lon@lpl.arizona.edu).

J. S. Halekas, D. L. Mitchell, and R. P. Lin, Space Sciences Laboratory, University of California, Berkeley, California 94720, USA.

M. Acuña, Goddard Space Flight Center, Greenbelt, Maryland 20771, USA.

A. B. Binder, Lunar Research Institute, Tucson, Arizona 85747, USA. 